\title{
Planes versus proyectos: Algunos problemas constitutivos del Urbanismo Moderno. Buenos Aires (1910-1936).
}

Alicia Novick

\section{Filiación}

Investigadora del Instituto de Arte Americano, Facultad de Arquitectura, Diseño y Urbanismo, Universidad de Buenos Aires.

\section{Resumen}

En este escrito el centro de interés reside en la relación que se establece entre las ideas globales sobre la ciudad y las propuestas de intervención. A diferencia de las argumentaciones tradicionales que aducen el escaso impacto de los planes en la constitución del espacio urbano, nos interesa mostrar que, además de dar cuenta de las formas de pensar y de actuar sobre la ciudad en un momento dado, inciden directamente en su constitución.

\section{Palabras clave}

Ciudad de Buenos Aires, Urbanismo Moderno, planes urbanos.

\begin{abstract}
In this paper the focus lies in the relationship established between global ideas about the city and the proposed intervention. Unlike the traditional arguments that claim the limited impact of plans in the constitution of urban space, we want to show that, in addition to account for the ways of thinking and acting on the city at any given time, directly affect their constitution.
\end{abstract}

\section{Key words}

City of Buenos Aires, Buenos Aires modernization, urban plans.

\section{Sumario}

Introducción

1.- El nuevo Plano, entre Proyecto y Plan

2.- Un Plan de proyectos

3.- El Plan en el proyecto

4.- Planes y Proyectos

Referencias 


\section{Introducción}

En el debate actual, muchos autores diferencian el proyecto urbano, del plan de urbanismo y el proyecto de arquitectura (1). Los defensores del proyecto urbano asocian el "clásico plan" a un producto técnico de alcance global que se perfila hacia el largo plazo, contrariamente al proyecto de arquitectura, cuya unidad lleva la impronta de un autor y cuyo impacto es delimitado en el tiempo y en el espacio. La noción de proyecto urbano se construye como una bisagra entre ambos extremos, que se presentan como pares polares estigmatizados. A diferencia de los "planes", el proyecto urbano daría forma al espacio sin intentar incidir sobre el conjunto de la ciudad y por oposición a los "proyectos de arquitectura", plantea otra vinculación con el tiempo (incorporándose a las largas temporalidades urbanas). Así, el proyecto urbano se presentaría como el último baluarte del urbanismo frente a la planificación "abstracta y managerial". Según otras opiniones, los "proyectos urbanos" son vértices de proyectos globales sobre la ciudad, consensuados socialmente mientras que para sus críticos manifiestan la legitimación de la especulación inmobiliaria frente a la carencia de un arbitraje público que garantice los equilibrios sociales y espaciales de la ciudad.

Las nociones de "proyecto" y "plan" tienen sus raíces en la Arquitectura. En su origen etimológico, que data del renacimiento, aluden a la geometría, a la representación gráfica- y refieren a la organización de una composición artística, en el espacio y en el tiempo, adecuando medios y fines. El objeto de la Arquitectura sería la elaboración del "proyecto", el del urbanismo, "el plan". La distinción entre "proyecto de arquitectura" y "plan" se funda en la multiplicidad de actores concernidos y en la consideración del tiempo y del espacio. Según Lacaze, proyecto alude a un: "procedimiento intelectual mediante el cual se propone una realización, justificando de antemano, por un conjunto de planos y de cálculos, la coherencia del objeto a construir y su adecuación al programa definido de antemano por el comitente". En tanto el Plan de urbanismo: "tiende a obedecer una lógica diferente de aquella del proyecto, marcando una interacción compleja entre decisiones públicas y elecciones efectuadas libremente por numerosos comitentes privados" (2).

¿Cuándo se origina la noción de proyecto urbano? Tal como lo recuerda F. Choay, "La aparición de términos nuevos o la difusión de antiguas palabras bajo nuevas acepciones marcan generalmente la emergencia de nuevas problemáticas" (3). Progettazione, urban design, proyecto urbano son, en efecto, términos acuñados en un escenario histórico preciso: el fin de la década del sesenta, cuando las propuestas del Movimiento Moderno deslizan desde la vanguardia hacia la experiencia evaluable. En esa instancia se revisan las relaciones entre la arquitectura y la ciudad (su contexto urbano construido, su medio social y su historia), traduciéndose en nuevos métodos de análisis y formulación de propuestas. Las investigaciones sobre el tipo y la morfología urbana ilustran estos nuevos enfoques que conciben una producción fragmentaria y acotada del espacio de la ciudad. Es ilustrativa, en este sentido, la denominación que utilizan los italianos para referirse al proyecto urbano: progettazione (4). El término intenta condensar en un solo procedimiento y en una expresión unitaria tres dimensiones del proceso de diseño: el diagnóstico, la concepción del proyecto y su implementación. 
Más tarde, a fines de los ochenta, el proyecto urbano se incorpora como una dimensión de la planificación estratégica. Esta perspectiva del planeamiento sustituye la idea de la gestión tecnocrática centralizada por procedimientos de gestión concertados entre los múltiples actores urbanos que intervienen en la producción de la ciudad. En una dinámica compleja, sobre la base de la identificación y selección de los múltiples proyectos sociales, espaciales, económicos, se va constituyendo un proyecto de ciudad sobre la base de escenarios alternativos para el futuro, abiertos y consensuados. Dentro de ese marco, "el proyecto urbano", deviene una intervención emblemática a los efectos del marketing urbano (5) y o un medio operatorio para formular el plan estratégico (6).

Desde lo anterior, es evidente que el proyecto urbano, la palabra, su definición y alcances son un producto reciente $-y$ no es posible asimilarlo a la "composición clásica"- aunque los efectos revival reivindiquen antiguos procedimientos. Sin embargo, la noción está atravesada con varios de los nudos problemáticos que la insertan dentro de los dilemas que el Urbanismo Moderno enfrentó a lo largo del siglo. En primer término, se plantean los conflictos entre la consideración del conjunto y la resolución de los problemas particulares. Tal como lo muestra la literatura, desde sus orígenes, el Urbanismo en tanto dimensión de la reforma social, como política pública moderna, intentó una acción sobre la globalidad de la ciudad para responder a problemas de diverso orden (sociales, espaciales, técnicos) conciliando los intereses privados y los colectivos. Sin embargo, los intentos de conciliación entre el espacio global de la ciudad y sus partes (que se dirimen también entre las ideas abstractas de planificación y el espacio construido; entre la "creación individual" y la "estética de conjunto"), no terminan de resolverse, ni en la concepción clásica - la construcción del conjunto sobre la relativa autonomía de los proyectos- ni en los planteos de la modernidad que intentan subordinar los proyectos a un planteo del conjunto -.

En segundo término, el diagnóstico - tributario de los métodos de la ciencia positiva intentaba proveer una evaluación de todos las dimensiones en juego para proponer instrumentos previsionales para la acción. Sin embargo, la conciliación compleja entre el diagnóstico preliminar (previsiones, cifras), las formas de acción propuestas (proyecto, intervención) marcan impasses, que Jean-Pierre Gaudin indicó con precisión en sus textos (7). Habitualmente el diagnóstico se efectúa desde los instrumentos disponibles.

Es en ese campo de tensiones constitutivas que nunca se resuelven, donde reside una de las claves para situar históricamente estos temas. En este trabajo, formularemos la hipótesis según la cual las tensiones que se establecen el plan y el proyecto - como nociones históricamente construidas - manifiestan algunos de los problemas centrales del urbanismo y permiten poner en perspectiva la reciente noción de proyecto urbano. Más allá de los ciclos que propone Collins (8), las oposiciones entre arte urbano y urbanismo del Movimiento Moderno, entre urbanismo "reglamentario" y "operacional", sostenemos que en cada escenario histórico, desde distintas perspectivas, se intenta resolver esos dilemas. 
En textos anteriores, mostramos que los proyectos locales que anteceden a los planes de los inicios de éste siglo, para los que se contrata a técnicos extranjeros, constituyen un campo de experimentación que conjuntamente con una progresiva institucionalización de la Universidad y la Administración Pública relativizaba el peso de las influencias extranjeras. En este texto, el centro de interés reside en la relación que se establece entre las ideas globales sobre la ciudad y las propuestas de intervención. A diferencia de las argumentaciones tradicionales que aducen el escaso impacto de los planes en la constitución del espacio urbano, nos interesa mostrar, que además de dar cuenta de las formas de pensar y de actuar sobre la ciudad en un momento dado, inciden directamente en su constitución.

Según la problemática esbozada, centraremos el análisis en tres escenarios históricos, coincidentes con tres documentos de urbanismo, cuya confección encargó la Municipalidad de la Ciudad de Buenos Aires durante las primeras décadas de este siglo. En una primera parte, se examina el Nuevo Plano (1907-1911) - a cargo del francés Bouvard y de un grupo de técnicos locales -. Coherente con una tradición decimonónica, intenta conciliar en el trazado, estética y razonamiento por funciones, en el ámbito de una idea de ciudad cerrada y jerarquizada. El plano se plantea entre las determinaciones formales del proyecto y aquellas indeterminaciones propias del plan moderno.

En un segunda parte, nos referiremos al Proyecto Orgánico de la Comisión de Estética Edilicia (1923-25). El documento, un plan de proyectos, refleja las tensiones entre los principios del arte urbano y los alcances del diagnóstico científico.

En una tercera parte se estudia la incorporación de la oficina técnica de urbanismo dentro de la administración municipal (1932). El intento frustrado de un Plan Regulador -s e efectúo un diagnóstico exhaustivo que no se plasmó en un documento pone en evidencia que ideas globales sobre la ciudad -formalizadas o no en planes- se dirimían por detrás de cada uno de los proyectos.

\section{1.- El nuevo Plano, entre Proyecto y Plan}

Hacia el fin de siglo, los problemas de la obra pública se tornan particularmente complejos en Buenos Aires, que en 1880 deviene Capital de la República. Sus amplios límites jurídicos delimitados en 1888, incluyen en el territorio de la Capital Federal amplios terrenos sin urbanizar y núcleos ocupados por antiguos pueblos que es preciso poner en comunicación. La gran ciudad recibe un importante cauce inmigratorio, que impulsa un crecimiento demográfico sostenido. Asimismo es sede de inversiones nacionales e internacionales en infraestructuras y servicios. Todos esos factores, aunados a la acumulación derivada de las grandes exportaciones de producción primaria genera una fuerte especulación en tierras e inmuebles. Las obras de gran escala, los edificios representativos del Estado Nacional y las múltiples iniciativas individuales, que la crisis del noventa no logra frenar completamente, se solapan con la complejidad de gestión que suscitan los procesos de modernización.

En efecto, desde fines del siglo pasado, los medios técnicos plantean la necesidad de controlar las modalidades de producción del espacio, tanto en el nivel de las Obras 
Públicas como de la construcción privada. En esta demanda, es clave el rol que les cabe a los profesionales, jóvenes egresados de las nuevas disciplinas que se van constituyendo en la Universidad, principalmente ingenieros e higienistas. Ellos toman a su cargo la resolución de diversos problemas del espacio urbano a medida que van ocupando espacios en las dependencias de la Administración que ellos mismos contribuyen a crear (10). En un primer momento la Sociedad Científica Argentina (11) se constituye en ámbito de reflexión. Allí, se debaten los temas de infraestructura, los relevamientos cartográficos, el trazado del catastro (12), los Reglamentos de Edificación (14), las técnicas constructivas (15), etc. Más tarde, estos principios serán puestos en práctica en las reparticiones nacionales y municipales. Por un lado, una amplia gama de reparticiones se ocupan de temas de saneamiento mientras la Oficina de Obras Públicas (reglamentada en 1891) reformula el Reglamento de Construcciones (aprobado en una primera versión de 1887), inicia las tareas de levantamiento catastral (1894), efectúa tareas de regularización (pavimentos, nivelación, alineamientos). Asimismo, en el nivel municipal, se fueron transformando las modalidades de control urbano - que se transfirieron de las Comisiones de Vecinos a las oficinas de la Administración municipal (16). Esa demanda de racionalización e "institucionalización" de los saberes sobre lo urbano se nutría de las múltiples experiencias europeas en planes de embellecimiento y extensión, vehiculizadas por medio de la bibliografía y el intercambio de profesionales.

En ese clima, después de concluir Plano Topográfico de la Ciudad en 1894, la Intendencia designó una Comisión para emprender el trazado definitivo de calles, plazas y avenidas, "de acuerdo al cual debía procederse en el futuro". El Plan, que se encarga en 1894, era imaginado como un instrumento de convergencia de todas las iniciativas públicas y privadas, un instrumento más en la amplia gama de herramientas utilizadas en la regulación del espacio urbano. Tal como se planteaba en la Revista de Ingeniería, en un texto que planteaba la necesidad de coordinación de todas las obras públicas: "si de una vez se formulara un plano regulador de las obras inherentes a un nuevo trazado del Municipio, a más de la homogeneidad y armonía de los proyectos, se conseguiría evitar tanto desacierto administrativo, que tantos prejuicios irroga a los intereses públicos" (17).

Para su confección, fue convocada una Comisión de notables con la finalidad de asesorar a la Intendencia en la toma de decisiones. Todos ellos eran funcionarios y miembros de la Sociedad Científica: el Director de la Oficina de Obras Públicas de la MCBA, Ing. Carlos M. Morales (ex-presidente de la Sociedad Científica Argentina), el Arq. Juan A. Buschiazzo (ex Director de Obras Públicas de Alvear y proyectista de la apertura de la Av. de Mayo), el Ing. Carlos Olivera (de la primera promoción local de ingenieros) y el agrimensor Eduardo Castex. El Plano de Mejoras (18), que recién se da a conocer en 1898, base del Plano de alineación de 1904, es un documento exclusivamente técnico que se complementaba con una amplia gama propuestas de saneamiento y regularización.

La resolución yuxtapuso dos niveles. Por un lado una matriz básica, la cuadrícula que permitía el control de la urbanización sobre el conjunto del municipio. La cuadrícula severamente cuestionada como morfología desde el arte urbano (19), es el medio que permite a la vez homogeneizar el espacio público, librar tierras al mercado y asegurar 
el rol de control de la Municipalidad. Dicho de otro modo, constituye la base que permite controlar el espacio urbanizado y a urbanizar (20). Superpuesta a dicha trama, un sistema de parques y una red jerarquizada de grandes avenidas ponía en relación la extensión de la ciudad, las estaciones de Ferrocarril y los grandes edificios públicos. Fue aprobado por el Concejo Deliberante el 21 de noviembre de 1898 pero nunca fue visado por el Congreso Nacional. La articulación que propuso el Plan de Mejoras de fin de siglo fue aun tosca, pero marcó las dimensiones sobre las que se trazarán la mayoría de las propuestas para el Centenario.

Desde una idea de ciudad cerrada y jerarquizada, el Plan de Mejoras propuso una red de parques, una Av. Costanera y un sistema de avenidas diagonales céntricas en torno de los dos principales espacios públicos: la Plaza de Mayo y la del Congreso. La red de parques y plazas es iniciada en la labor de Carlos Thays desde la Dirección de Parques y Paseos de la Municipalidad de Buenos Aires. Este conjunto, fue completado paulatinamente durante las primeras décadas del siglo por dicha dirección y consagrado en los veinte, con los aportes de Forestier, desde la Comisión de Estética Edilicia (21).

La frontera entre el territorio urbano y los lejanos suburbios se aseguró por un ambicioso proyecto de "ronda". Una avenida Costanera, continuada por la rectificación del Riachuelo y una calle Periférica, eran la materialización de los límites establecidos para la Capital de 1886. Propuesta por el Departamento de Obras Públicas Municipal en 1896, reinterpretaba la imagen de los boulevards europeos. Tal como afirmaban sus autores: "(El camino de la ribera y la Av. de circunvalación) separará(n) el Municipio de la Capital de la provincia de Buenos Aires, y, finalmente, cerrando este hermoso circuito se tendrá el paseo proyectado a lo largo del Riachuelo que se prolongará hasta Barracas. Así, encerrada en este hermoso marco, con sus grandes parques y paseos, sus amplias avenidas, sus grandes edificios públicos y privados y su población, que irá siempre en aumento, atraída por este gran centro, se puede tener idea de lo que será la que ya es hoy primera ciudad de América" (22).

"La primera ciudad de América", era también el escenario para el despliegue de los edificios públicos que representaban al estado Nacional (23). El Congreso, al igual que las otras construcciones monumentales - como el Correo, los Tribunales, el Teatro Colón, la red de escuelas públicas- relacionados entre sí y con la Plaza de Mayo. Las diagonales irradiantes desde la Plaza de Mayo y el Congreso, el monumental eje de la Av. de Mayo -primera apertura "hausmaniana" iniciada en 1886- y la Av. Norte-Sur (que unía las estaciones de Ferrocarril) inauguran el esquema básico incluido en el Plano de Mejoras que será objeto de controvertidos debates durante las primeras décadas del siglo. Más allá que el influjo del modelo parisien que deja su impronta en los debates de las diagonales, interesa marcar dos cuestiones centrales, vinculadas a la problemática tratada. En primer lugar, estos proyectos marcan una importante oposición entre el embellecimiento del centro y la extensión, que se plantea contemporáneamente. En segundo lugar, abren la participación a los arquitectos a los temas urbanos, hegemonizados hasta ese momento por los ingenieros y los higienistas.

En efecto, desde las últimas décadas del siglo XIX, los higienistas, los reformadores sociales y los nuevos partidos políticos fueron desplazando sus preocupaciones desde 
los "conventillos" hacia los bordes de la ciudad, que sin servicios y sin infraestructuras se constituyeron como espacio de localización de los sectores de menores ingresos. El crecimiento de la ciudad lentamente se construye en tanto problema social. En ese momento se polarizan las opiniones entre aquellos que priorizan el embellecimiento céntrico (con justificación de circulación e higiene) de los que se preocupan por el acondicionamiento de los barrios suburbanos. Por ejemplo, la propuesta de apertura de la Avenida Norte Sur, pensada como una vía de jerarquización y circulación, se asociaba con las expropiaciones de la Av. de Mayo y condensaba múltiples críticas: "Crear avenidas en el centro de la ciudad, procediendo a la expropiación de centenares de propiedades es empezar por donde debe concluirse, cuando la capital está casi incomunicada con los centros suburbanos que la rodean a causa de los caminos que son una verdadera vergüenza (...)" (24). Los conflictos entre la necesidad de controlar problemas del crecimiento y el embellecimiento del centro, son las dos caras tradicionales de la modernización decimonónica que se intenta resolver. "Crear una avenida grandiosa - cuando no tenemos hospitales, ni asilos, ni todas las calles abiertas, cuando tenemos todavía barrios populosos alumbrados con kerosene, cuando no tenemos como destruir las basuras de la ciudad sino empleando medios primitivos, cuando nuestros mataderos son un foco de infección (...) París tenía todo eso y mucho más cuando empezó a realizar sus proyectos, nosotros deberíamos hacer eso mismo" (25). En las antiguas ciudades europeas se tiende a "eventrar" y funcionalizar el centro (a la manera hausmaniana), o, a "proteger" los antiguos centros - condensadores de los valores tradicionales, históricos - urbanizando el suburbio a la manera del ensanche de Barcelona o del Ring vienés. En Buenos Aires el conflicto era doble, porque se necesitaba construir a la vez la ciudad "vieja" - con edificios y espacios públicos - y "la nueva" - la urbanización de los sectores más alejados, "los barrios suburbanos" que requerían de la intervención pública. Planes y proyectos debían resolver ese conflicto. En ese momento, aunque se insinúan senderos de convergencia, se tratan aún en esferas diferenciadas los temas de ingenieros e ingenieros sanitarios -preocupados por asegurar las infraestructuras-, de los higienistas y reformadores - que plantean soluciones para la vivienda obrera - y de arquitectos ocupados en los trazados y el embellecimiento del centro.

Los textos de Camillo Sitte, y más globalmente el ideario del arte urbano consagran las figuras de los arquitectos como protagonistas de los planes de remodelación. El "plan de urbanismo que produce un efecto estético es una obra de arte y no un simple asunto administrativo" (26). Desde esa división de tareas ideal, los funcionarios municipales tomarían a su cargo los problemas de la extensión, las comunicaciones y la adecuación del espacio a las nuevas actividades. Por su parte, los artistas asegurarían el embellecimiento público por medio de los trazados, edificios y monumentos, que desempeñan un rol pedagógico en el seno de la comunidad. En ese sentido, en las vísperas del Centenario, desde sus recientemente constituídas corporaciones profesionales solicitaban Concursos para la confección de planes de embellecimiento al tiempo que criticaban la acción de los funcionarios municipales (27). La Intendencia, en el contexto de la preparación de las obras para el Centenario de la Emancipación, que se preparan para 1910, resuelve contratar directamente a Joseph Bouvard, Jefe de los Trabajos Públicos de París (28). 
¿Cómo resuelve los problemas de Buenos Aires el Nuevo Plano de 1909, que confecciona el francés Bouvard conjuntamente con una Comisión de Técnicos?

El objetivo inicial, tal como fue enunciado en el documento, era el de una puesta en coherencia: "ante la diversidad de proyectos que se han hecho públicos (hace) de indiscutible utilidad hacer un estudio de conjunto" (29). En efecto, el documento arbitró entre las propuestas en danza. Bouvard transmitió su experiencia en la confección de la memoria descriptiva, en su dominio de la bibliografía y en las tomas de partido globales, que le permitieron presentar de una nueva forma las propuestas urbanísticas. Las menciones eruditas de Henard - en temas de circulación - de Buls en referencia a la estética urbana - y de Forestier - como experto en sistemas de parques - otorga la "fundamentación teórica" a la propuesta. A diferencia del Plano de Mejoras, apunta a organizar un razonamiento global, aunque el planteo es aún fragmentario y se apoya sobre los grandes proyectos en tanto núcleos de la composición. De hecho, la Comisión del Nuevo Plano no tuvo competencias para resolver la localización de los edificios públicos, las estaciones de Ferrocarril enunciados como problema- ni los temas de vivienda y de infraestructuras debatidos en otros ámbitos.

El proyecto espacial, se presentó en dos versiones. El primer plano es una síntesis sobre fondo blanco con las principales líneas del proyecto; el segundo es un plano topográfico de la ciudad cubierto con la cuadrícula, sobre el cual se imprimen los proyectos de red vial, plazas y parques. En los límites del arte urbano, en términos del tratado de Cloquet (30): "el trazado del plan de una ciudad comprende la delimitación de sus calles y sus plazas". Simultáneamente, se diseñaban los proyectos para la plaza del Congreso, la explanada abierta para la Plaza de Mayo, el Balneario, el trazado de las plazas y jardines como elementos relativamente autónomos. En términos de Cornelius Gurlit: los objetos del arquitecto "eran las notas (hechos singulares) del ritmo de la melodía urbana (el conjunto)". Los planos, los trazados del arte urbano debían poner en relación "estas notas".

La organización general se estructuró con una descripción crítica de lo existente y un listado de las transformaciones a efectuar en las Plazas (Plazas públicas, Parques y Jardines), el sistema vial (Vías Públicas a crearse, continuarse y ensancharse, Vías irradiantes o concéntricas, oblicuas o diagonales, Vías de Prolongación y de unión, Vías o parte de las vías actuales a ensancharse) y en los puntos de articulación (encrucijadas, estaciones de Ferrocarriles, edificios Públicos). La única idea fuerza del partido, tributaria de la experiencia del francés, fue la recuperación de la relación de la ciudad con el río. El proyecto de Avenida periférica - Av. Costanera, planteada por el Departamento de Obras públicas y clave del Plano de Mejoras, se amplió en la intención de recuperar el río. "Las disposiciones adoptadas hasta ahora, y, en particular en la parte más densa de la ciudad, han hecho a un lado los hermosos aspectos del incomparable río que la bordea" (31).

El diagnóstico sobre los problemas de la ciudad fue breve y cada de los problemas identificados en la ciudad existente se vinculaba con un elemento compositivo disponible. Así la falta de higiene (el aprovechamiento del aire puro, la regulación del movimiento), la "utilización social" (punto de reunión, de descanso, sitio agradable, 
lugar de fiestas y ceremonias); de "estética" (ubicación apropiada para monumento) se resuelve con los diseños de parques y las plazas. Para resolver las cuestiones de circulación, se efectuó una acérrima crítica de los perjuicios de la cuadrícula que "impide las comunicaciones directas y la comunicación fluida entre diversos puntos"; "marca una difícil distribución de los edificios", "una ausencia de perspectivas pintorescas y atrayentes". Frente a este panorama, se propuso "un sistema" de calles, concéntricas, irradiantes de forma oblicua o diagonales, que tienden a "descongestionar el centro comercial y financiero" y " a mejorar sensiblemente la estética de la ciudad" (32).

El Nuevo Plano, fue un producto de transición entre "un proyecto" y "un plan de urbanismo". Fue un proyecto, porque se propuso llevar a cabo con coherencia las transformaciones de la ciudad como objeto delimitado y autores precisos. La justificación (aun no se trata de un diagnóstico sistemático) se efectúa sobre los problemas de la ciudad - circulación, rentabilidad económica, higiene, vida social- en correlato con los instrumentos compositivos utilizados. Pero al mismo tiempo, consideran indeterminaciones en el tiempo "es preciso preocuparse del porvenir $y$ trazar para ello, hoy una norma de conducta, un programa fijo en la vía de progreso", en el espacio (la trama ortogonal que se extiende, se delimitan los lotes para construir por los particulares, los sitios donde los artistas deben desplegar su arte) y en la gestión se consideró el rol de la Administración Pública para llevar a cabo la tarea. Según la definición dada el "plan" consistía en "reglas generales que han de seguir en adelante las autoridades y a las cuales será preciso ajustarse en la ejecución de las obras que se realicen de acuerdo con las circunstancias y recursos disponibles" (33).

Globalmente, el documento se caracterizó por formular nuevos instrumentos operativos, más que en propuestas novedosas de transformación urbana. En la elaboración del Plan, los proyectos pasan a formar parte de un esquema sistematizado de los cambios de la ciudad. De hecho, con posterioridad, se iniciará la construcción del balneario, la creación de nuevas plazas, se aprobarán en el Congreso los proyectos de avenida Norte - Sur y Avenidas diagonales (1912), etc. Aunque el Nuevo Plano no se lleve a cabo de toutes piéces, muchos de los proyectos planteados se instalaron en el debate público y se fueron concretando a lo largo del siglo.

\section{2.- Un Plan de proyectos}

En 1923, el Int. Dr. Carlos Noel convoca una Comisión de Estética Edilicia (CEE), con el objetivo de trazar el estudio de las reformas de la ciudad: El Proyecto Orgánico para la Urbanización del Municipio. El Plano Regulador y de Reforma de la Capital Federal (34), publicado dos años después.

Al igual que el Nuevo Plano, el documento se propone conciliar una amplia gama de proyectos - anteriores y nuevos-, como una etapa previa para la realización de un verdadero plan regulador, cuyos alcances se precisan en un último capítulo. A diferencia de él, es el primer documento local, que intenta responder a las ópticas del urbanismo Moderno, retomando sus temas - problema y con una bibliografía actualizada. Tal como se lee en el título, no se trata de "un plano" ligado aún a una imagen cartográfica, sino de un "proyecto orgánico". Este refiere al organismo de la 
ciudad, para el cual se diseña una propuesta "moderna, razonada y metódica" para su reforma y regulación. Es un documento que precisa intenciones globales sobre la ciudad y su vida colectiva. La "totalidad" es social, espacial, funcional y apunta a la comunidad. "El plano de una ciudad es la expresión de una vida colectiva" y la ciudad, pues, no solo debe llenar una finalidad material de bienestar común sino invitar a sus habitantes a realizar un alto propósito" (35).

El objetivo central de la CEE es re-equilibrar la estructura urbana, de Buenos Aires, transformada (y deformada) por la extensión y el crecimiento. Desde esa situación, prevalece la necesidad de descubrir y conservar la identidad de la ciudad asegurando el sistema urbano. La idea de "sistema", - es entendida como una relación entre las partes y el conjunto: "El orden obtenido por la clasificación de las funciones de la ciudad, permite al urbanista dar a cada elemento el desarrollo real que merece y ellos son los encargados de construir el conjunto del Plan orgánico" (36).

A diferencia del Centenario, en la década del veinte la necesidad de un plan, para la coordinación global, responde a una demanda que comienza a formularse dentro de los medios técnicos y desde las filas de socialistas y reformadores sociales. En efecto, entre el Centenario y los años veinte, el foco de los debates se fue desplazando del embellecimiento a los problemas de la vivienda obrera. En 1912, la Ley Sáenz Peña de sufragio universal amplió la participación incorporando a los partidos políticos que, tras la crisis del '90, representan a las nuevos grupos emergentes de la inmigración. Nuevos proyectos relacionados con las condiciones de vida populares entraron en juego conjuntamente con la creación de múltiples Asociaciones que presionaban sobre las autoridades. Luego de los incipientes proyectos y realizaciones de los primeros años del siglo, la constitución de la Comisión de Casas Baratas (1915), los debates del Congreso de la Mutualidad (1918), de la Cooperación (1919), de la Habitación (1920) organizados por el Museo Social Argentino, le otorgaban un rol significativo a los problemas espaciales y sociales de la ciudad. Las conclusiones del Congreso de la Habitación de 1920, inspirados en el evento homónimo realizado en Londres ese mismo año, sintetizaban las preocupaciones: la necesidad de trazar planes, resolver el problema de la vivienda y, más globalmente controlar y racionalizar la producción del espacio urbano. Esas conclusiones fueron elaboradas durante una etapa afectada profundamente por la crisis de la posguerra, cuando los problemas de abastecimiento, afectaban las condiciones de vida en el Buenos Aires de la primera posguerra.

Tres años después, una mejor situación financiera y un cambio presidencial permitieron otorgar un nuevo impulso a las grandes obras públicas, que como en el Centenario, requerían de una puesta en coherencia. En 1923, el Intendente reunió la Comisión de Estética Edilicia (Intendencia, 1925). Según el documento inicial, fueron convocados los "elementos más autorizados de los poderes públicos encargados de vigilar la construcción de los elementos nacionales, las obras públicas a erigirse en la Capital Federal, que por su intermedio han de ajustarse al plan general(...)" (37). Los miembros de la Comisión fueron seleccionados entre las principales instituciones públicas con competencias sobre proyectos: el Arq. René Karman, -arquitecto francés que desde 1912 es uno de los jefes de Taller de la Facultad de Arquitectura-; el Arq. Carlos Morra -Presidente de la Sociedad Central de Arquitectos-, Sebastián Ghigliazza, Director del Ministerio de Obras Públicas de la Nación, representante del organismo 
nacional, ocupado en los grandes proyectos edilicios.; el Arq. Martín Noel, Presidente de la Comisión Nacional de Bellas Artes, el Ing. Victor Spotta, Director General del Departamento de Obras Públicas de la MCBA. La secretaría fue asumida por Emilio Ravignani, historiador y Secretario de Hacienda de la Municipalidad en tanto la resolución de espacios verdes y libres fue encomendado al francés Jean-Claude Forestier.

Los debates entre embellecimiento céntrico vs. equipamiento suburbano, problema constitutivo del urbanismo persiste. En ese sentido, se plantea la necesidad de descentralizar la ciudad, indirectamente por medio de la zonificación y las normas: directamente por las propuestas de "Barrios obreros, jardines y embellecimiento suburbano", una serie de centros cívicos y un sistema de espacios verdes y libres de carácter metropolitano. El rol de Forestier consistió en analizar, los proyectos de un empréstito aprobado en 1923, para construir la Av. Costanera, la ampliación del balneario, el mejoramiento de plazas, parques de ejercicios físicos, colonias de vacaciones, sistemas metropolitanos de parques. El francés planteó por primera vez en Buenos Aires, la necesidad de considerar el espacio extra-muros:. "los numerosos pueblos que circundan nuestra urbe, ... han de contemplar su posible anexión al ejido de la Capital, puesto, que, situados a lo largo de su cinturón urbano, están llamados a compartir su porvenir y ser en el presente fuentes de abastecimiento y tributarios de los servicios urbanos de la Capital federal" (38).

Se vislumbra, pese a un tratamiento algo rudimentario, el interés en examinar las tendencias para construir las leyes de evolución de Buenos Aires. El Proyecto Orgánico da cuenta de la evolución histórica de la ciudad, efectúa estudios estadísticos de población y edificación, de abastecimiento, tratamiento de residuos y de hospitales. No obstante, los estudios diagnósticos incluidos apuntan a dos situaciones contrastadas. Se plantean como justificaciones de las decisiones de proyecto adoptadas. O son análisis de carácter específico, sin vinculación con lo propuesto. Mencionemos a modo de ilustración que el análisis demográfico no se corresponde con la zonificación. De igual modo, el estudio de la historia de la ciudad es una argumentación que legitima los espacios a intervenir.

El centro de la propuesta de la CEE, consistió en reunir y analizar la factibilidad de los proyectos desde premisas de partido globales:" (...) para preparar el vasto plan de obras edilicias que propone, no ha pretendido indicar o formular nuevas soluciones, prescindiendo de lo que antes se hubiera estudiado o propuesto. Por el contrario, ha tomado especial empeño en recopilar antecedentes y estudios anteriores, a fin de compulsar opiniones, concretar observaciones y estudiar las diversas soluciones anteriormente propuestas para resolver este importante asunto" (39). Desde ese objetivo, se estudia la restructuración global de la red vial (Av. periférica, avenida de norte-sur, Av. Costanera), los accesos a la ciudad (Plazas de las estaciones ferroviarias: Plaza Constitución, Plaza Once de septiembre, Plaza de Retiro); los espacios cívicos (Plaza del congreso, Plaza de mayo) y de los centros para la expansión (Plaza de Italia, Parque Centenario), vinculándoles entre sí y reorganizando el conjunto de la ciudad. El diseño de estos sitios es evaluado en su diseño y en sus relaciones: "no (se) ha abordado el estudio definitivo de ninguno de los anteproyectos que propone, pues se considera que ese trabajo debe ser objeto de un meditado y largo 
estudio, que no encuadra dentro del carácter de un proyecto general de obras, como es el que se le ha encomendado preparar" (40).

En el análisis y elaboración de las propuestas le cupo especial protagonismo a René Karman - académico francés, profesor de Taller de la Facultad de Arquitectura de la Universidad de Buenos Aires- quien dirigió el equipo de proyectos. Los referentes directos fueron la city beautiful y del manual de civic art de Hegemann y Peets, que se incluyen en la bibliografía y otorgaron el sustento teórico de la propuesta. Las opciones de arquitectura, vinculadas a la arquitectura neocolonial, tendencia que encabeza Martín Noel, el hermano del Intendente. Existe un intento de considerar las funciones, la racionalidad compositiva y el respeto por las tradiciones, desde una búsqueda identitaria, rechazando los cánones estéticos de la modernidad (41). Los alcances que otorgan a la "estética", se vincula con el rol pedagógico y de valorización del pasado como fundamentales para la comunidad. "El ideal de un programa social superior es el que devolverá a las ciudades modernas la belleza perdida. Solo ciertos estados sociales provocaron la creación de los hermosos ejemplos del pasado" (42).

Un intento de abandonar esos criterios, fue propuesto contemporáneamente por dos jóvenes arquitectos, que vuelven de su iniciático viaje a Europa. Alberto Prebisch y Ernesto Vautier, admiradores de los protagonistas del Movimiento moderno, presentan en 1924 un proyecto urbanístico, inspirado en la ciudad Industrial de Tony Garnier. Sus láminas mostraban un conjunto zonificado, con construcciones de hormigón y una graficación austera. "El problema, para ser bien planteado, requiere ante todo, apartar en absoluto toda consideración sentimental, el conocimiento perfecto de las diferentes piezas que han de coordinarse para integrar el inmenso mecanismo que constituye la ciudad" Cada pieza debe desempeñar estrictamente su función en el conjunto" (43). El proyecto in toto de Prebisch y Vautier, recupera una experimentación anterior en vivienda obrera e introducen por primera vez la idea de un plan que subordina la arquitectura al conjunto. "la arquitectura pierde todo significado como expresión individual de una idea estética. Pero se convierte en la fiel expresión de un sentir colectivo" (44). Su Ensayo de Estética Contemporánea marca la emergencia de una corriente, que auspiciará las célebres conferencias de Le Corbusier en 1929.

A pesar de las múltiples críticas que la literatura de la época y la historiografía le formulan al documento, es importante puntualizar que éste se concibe a sí mismo como una etapa preliminar para la confección de un plan. El Capítulo final, propone la necesidad de constituir una Comisión ad- hoc para confeccionar un Plan y de Reglamentos que asegure la coherencia global de la ciudad (45). "El crecimiento de la Capital, no puede, en adelante, quedar librado al azar, y, una vigilante atención debe en todas partes como en todos los momentos, aplicarse como una constante voluntad de subordinar todos los casos particulares al concepto general del conjunto de la ciudad". El Plan, presentado como una necesidad imperativa, debería " asegurar la unidad y defensa de las ideas emitidas como la perseverancia de la prosecución de obras y proyectos (...), fomentar por la propaganda el interés público a fin de que su obra sea apoyada por todos. Es decir debe realizar, en completa armonía con la opinión pública esta obra de carácter esencialmente colectivo" (46). 
El Proyecto Orgánico recupera los proyectos anteriores, pone el énfasis en la composición grandes conjuntos edilicios como referentes para cualificar una ciudad que crecía. Sin embargo, los proyectos de plazas y centros cívicos, las redes de viaria y de espacios verdes apuntan a constituir sistemas estructuradores de la extensión y complejidad metropolitana. Las propuestas incluyen temáticas nuevas (aunque sin desarrollar) como viviendas obreras, problemas suburbanos, resolución de temas técnicos, que lo diferencia del Nuevo Plano del Centenario. No obstante se instala el conflicto entre el todo social, técnico, cultural - de lo cual daba cuenta el diagnóstico- y el carácter de las soluciones espaciales adoptadas.

\section{3.- EI Plan en el proyecto}

Las ideas del Urbanismo Moderno están ya firmente instaladas en el debate público de Buenos Aires, en la década del treinta. Las experiencias de los veinte, el Plan del 25, los Congresos, las visitas de profesionales de prestigio como Jaussély, Le Corbusier y Hegemann, asi como la difusión encarada por las publicaciones de las corporaciones profesionales y civiles contribuyeron a establecer mediaciones entre los ámbitos profesionales y el gran público y a obtener amplio consenso.

Con posterioridad a la convocatoria del Plan de la Comisión de Estética Edilicia de 1925, tres debates importantes en el Concejo Deliberante de la Municipalidad de Buenos Aires, muestran líneas de consenso en los medios legislativos. Antes de finalizar la confección del Proyecto Orgánico, una propuesta de 1924, brega por una consideración de los componentes higiénicos y técnicos, en abierta crítica a los planteos excesivamente "estéticos" que le imprimen al Plan, cuando recién aún se encuentra en vías de elaboración. En 1929 un socialista encabeza una solicitud para la confección de un Plan Regulador, cuyo propósito es resolver los desequilibrios sociales en la ciudad. En tanto, la tercera presentación, en 1932, con posterioridad al restablecimiento de la democracia después del golpe militar de 1930, manifiesta la urgente necesidad de elaborar un Plan Regulador y de instalar una Oficina técnica.

El mismo año, el Municipio crea la Oficina del Plan de Urbanización, cuya dirección queda cargo del Ingeniero-urbanista Carlos María Della Paolera, cuya trayectoria esta estrechamente ligada a la evolución del debate urbanístico porteño. El primer texto publicado por DP trata sobre estética urbana. Cuatro años después, en 1920, publica en la misma revista un largo informe sobre del Plan Regulador de Paris, y ese mismo año interviene en el Congreso de la Habitación, donde presenta con una propuesta de reglamentación profesional. Más tarde, durante su estadía en Francia (1924-27), envia artículos al diario La Nación, participando a distancia en la difusión de las ideas locales, mientras terminaba su estudios de doctorado. Su tésis, bajo la dirección de Marcel Poète, presenta una evolución urbanística de la ciudad (47), como base para la formulación de un Plan Regulador para Buenos Aires. Este trabajo seminal provee las bases de su actuación en los años treinta, en que deviene el brazo ejecutor de las estrategias de modernizadoras del Intendente de Vedia y Mitre, en un contexto de represión política y social (la literatura denomina "década infame" a este período de la historia argentina, cuando un gobierno elegido por sufragio fraudulento sucede al golpe militar de 1930). 


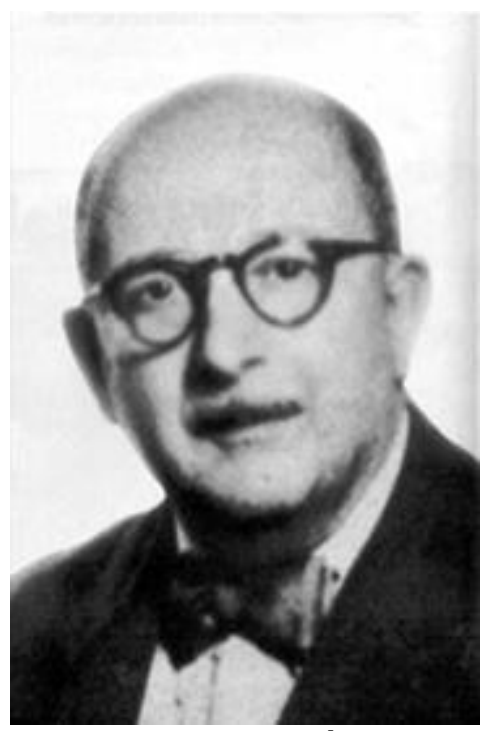

Ing. Urbanista Carlos María Della Paolera

Desde la Oficina Municipal, se plantea un amplio programa de acción para la urbanización regional. A los efectos de coordinar: problemas de circulación (red ferroviaria y caminera de la aglomeración bonaerense), redes de ciudades (la organización de las localidades suburbanas en función de la ciudad núcleo); de temas habitacionales (posible instalación de barriadas económicas en zonas propicias de los alrededores de la capital); espacios libres y verdes (creación, fuera y dentro del municipio zonas y reservas de espacios para el sistema de parques regionales) y localizaciones industriales, etc. Sin embargo, los logros son extremadamente restringidos. Si bien se emprenden obras metropolitanas (como los puentes que comunican con la provincia, a cargo del Ministerio de Obras Públicas), no se logra la deseada "coordinación metropolitana" (en Buenos Aires dichos organismos nunca pudieron consolidarse), los estudios preliminares de zonning recién tomaran cuerpo en el Código de Edificación de 1944, en tanto los emprendimientos sociales son casi inexistentes en el área de la Capital.

Conceptualmente, el programa responde paso por paso a las premisas instituidas por el urbanismo "científico", preconizadas por los trabajos de Agache, Gréber y Agustin Rey, autores de los tratados de mayor difusión y profesores del Instituto de Urbanismo, donde della Paolera sigue sus cursos de posgrado. La oficina de Urbanización, tiene un programa de trabajo claro, inspirado en el modelo médico. Según la metáfora orgánica, la ciudad es un "organismo vivo en un medio que reacciona bajo la acción de agentes externos e internos...". El urbanista es el médico, que efectúa el diagnóstico en base de la historia clínica - evolución urbanística- y concibe un tratamiento en el cual, ciertamente, no deben estar ausentes las consideraciones de la Estética. La meta es el organismo sano, modelo ideal al que se debe arribar mediante la conjunción del "arte" y de la "ciencia". 
Desde esas premisas, se desprende una metodología de trabajo precisa, que adopta la Oficina. En primer término, estudiar la evolución de la ciudad (la anatomía, que se pone en paralelo con la historia clínica). Para cumplir ese objetivo se desarrollan exhaustivamente los estudios diagnósticos - medio geográfico, trabajos históricos, estudios de humos - que hegemonizaban el cuerpo principal de la tesis de della Paolera-. En segundo término, elaborar los estudios estadísticos (la fisiología, que refiere a la constitución de un programa). El análisis censal y los estudios de la evolución del volumen construido, de las actividades económicas y comerciales dieron origen a numerosos documentos de trabajo. Como tercer punto, se procede a las propuestas espaciales - incluidas en el "arte urbano" (la clínica médica quirúrgica, plantea la necesidad de la intervención).

El papel que le cabe al expediente urbano, es introducir la "cientificidad" - en el análisis de los datos y en la clasificación de fenómenos, para fundamentar los principios que guían la acción. El "arte urbano", - es según ese planteo el resultado de un diagnóstico y el último eslabón de la amplia gama de estudios previos pero también el lugar donde se despliega "la intuición" que marca el diseño de los trazados y los proyectos.

Della Paolera intenta precisar esta particularidad y diferenciarse de planteos anteriores: "un plano es "una representación gráfica, agradablemente coloreada, en la que figuran todas las transformaciones proyectadas para una ciudad existente o todas las estructuras previstas para una ciudad a crearse", contrariamente, un plan (regulador) debe comprender a muchos "planos" y toda clase de representaciones de la ciudad y sus problemas...la propaganda, la enseñanza y la lucha urbanística y debe amoldarse a la metamorfosis rápida de los complejos vitales de la urbe..." (48). Desde esa acepción restrictiva, el "plan" sería el resultado de estudios permanentes, del expediente urbano, de los profesionales y la propaganda en tanto "el plano", próximo a la carta geográfica, sería una propuesta formal (que engloba a los planos de embellecimiento y extensión del período anterior). En ese contexto, el rol del "proyecto" es una intervención totalmente tributaria de un programa que contempla la ciudad en su conjunto.

La Oficina, a pesar de proponer sus programas de acción y completar una amplia gama de estudios diagnósticos, que se presentan en exposiciones, eventos científicos y cursos universitarios, no logra elaborar un Plan Regulador oficial. No existe ni siquiera un documento programático que incluya todas las propuestas de acción, jerarquizadas y precisas. Sobre el final de la gestión se elabora un texto (49), que intenta otorgar una coherencia entre las obras planteadas, pero es una suerte de justificación ex.post que pone en relación la fragmentaria acción llevada a cabo. Las razones para ello pueden ser múltiples -la dimensión restringida de la Oficina, su rol subordinado a los designios del Intendente; pero sin examinar sus causas (que exceden a este texto) (50) es pertinente analizar sus consecuencias. Los alcances del "plan ausente", se dirimen en los debates que suscitan los mega-proyectos, donde se vislumbran los impasses entre diagnóstico y propuesta; entre las ideas globales sobre la ciudad y las intervenciones fragmentarias. 
Una ilustración paradigmática de estos problemas, puede examinarse en la competencia entre proyectos que se planteó en cuanto a la elección del trazado de la Avenida 9 de Julio. Esta apertura, es producto de un antiguo proyecto de 1895. El eje este-oeste de la Av. de Mayo, se complementaría con un eje Norte Sur, uno de los puntos del debate con las diagonales de la inflexión del siglo. Su trazado original, que preveía una calle de 33m. de ancho, se aprobó en enero de 1912 (Ley 8855), con posterioridad al Nuevo Plano. El Plan de 1925, desde su prioridad por la descentralización de los centros cívicos, la integraba con las diagonales Norte y Sur configurando los extremos de la base del triángulo con dos hitos monumentales.

Della Paolera confecciona dos proyectos sucesivos $(1933,1937)$, que en lugar de los $33 \mathrm{~m}$. de ancho proponen liberar una manzana completa de $100 \mathrm{~m}$ para crear una "avenida parque" sin edificación. La diferencia entre ambos reside en que el primero se organiza alrededor de una calle de circulación rápida subterránea en tanto que el segundo la mantiene a nivel evitando los cruces con el subterráneo existente. En 1934 plantea una variante que consiste en la creación de un centro de Edificios de Gobierno en el cruce con la Av. de Mayo y dos remates monumentales, similares a los que se plantearan en el Plan del 25. Este último proyecto dialoga abiertamente con otras propuestas contemporáneas. Fermín Bereterbide (51) retoma en 1932 el proyecto original de una Av. de $33 \mathrm{~m}$ flanqueada por una faja continua de edificios -viviendasde igual altura (8 pisos), que articula centros cívicos. Globalmente este proyecto, replica la resolución de Eliel Saarinen para Chicago (1922) (52) y la combina con edificios de vivienda. Por otro lado, Ernesto Vautier -que elaborara junto con Prebisch la "Ciudad Azucarera" en 1924, reformula dicha idea (53) un año después planteando un basamento continuo de comercios y jardines de dos pisos y concentrando la edificación en una o dos torres - supermanzanas- que eliminan la distribución de los lotes y manzanas tradicionales. Ambos proyectos prevén una viaria subterránea (solución técnica hegemónica en los treinta (54)). Estos proyectos controvertidos -a los que podrían sumarse la "Monumentalización Funcional" de Ángel Guido (55) o la resolución de los ingenieros del Ministerio de Obras Públicas-, muestran la intensa experimentación urbanística de la época, cuando la rivalidad entre proyectos viene aparejada de una lucha por el dominio de espacios profesionales e institucionales. No obstante, no es difícil identificar los modelos urbanos que inspiran a cada proyectista.

"La ciudad vertical", "las propuestas habitacionales de las municipalidades reformistas", son algunos de los referentes. Pero no se trata solamente de una referencia a modelos, usual en la mímesis del diseño de arquitectura. Los proyectos presentados pueden considerarse como fragmentos de ideas globales de la ciudad en su conjunto, sino que son fragmentos de una ciudad imaginada en su conjunto.

El triunfo del proyecto Della Paolera es haber logrado imponer la configuración de park-way, sin edificación (56), sin arquitecturas; un pulmón urbano opuesto a las versiones que defendían sus contrincantes Beretervide y Vautier, coherente con la operación de desplazamiento del centro - que desde el siglo pasado se localizaba en el eje Plaza de Mayo- Avenida de Mayo- Congreso- hacia el Norte. Su propuesta será el blanco de numerosas críticas. 
Desde el punto de vista financiero se objeta la pérdida de la potencial renta para el Municipio que representarían los terrenos sobrantes en el proyecto original de 30 metros. Desde lo urbanístico, la idea de una avenida parque en el centro urbano es duramente criticada por los sectores más ortodoxos de los arquitectos del Movimiento Moderno. En tanto el proceso de toma de decisiones que precedió a su apertura tuvo ribetes tan conflictivos en los espacios de debate político que, aún cuando las obras ya se habían comenzado, se continuaban formulando proyectos alternativos. 


\section{REVISTA DE URBANISMO \\ ISSN 0717-5051}

http://revistaurbanismo.uchile.cl
Planes versus proyectos: Algunos problemas constitutivos del Urbanismo Moderno. Buenos Aires (1910-1936).

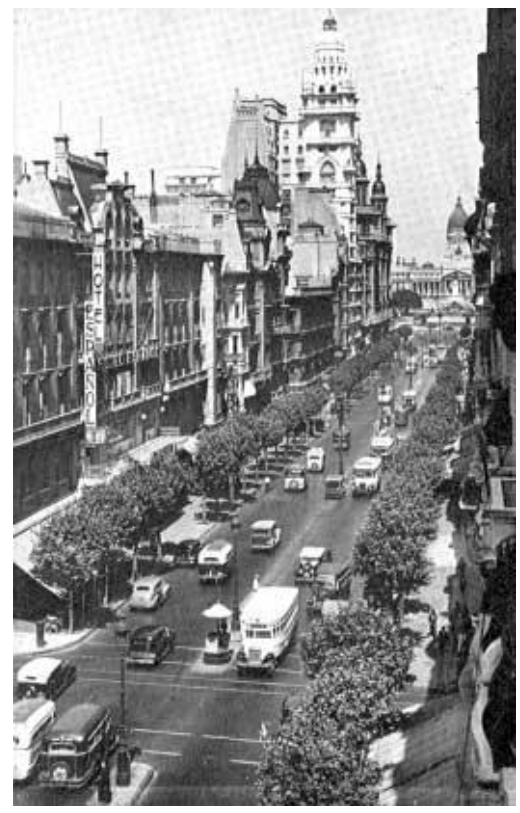

Buenos Aires, Avenida de Mayo. (Supra) vista hacia el Congreso en la actualidad, y (infra) vistas en el pasado.

Tarjetas Postales en archivo M.I.P.R. Reproducción parcial en blanco y negro con fines estrictamente culturales. 
Pero es interesante analizar cómo se justificó la propuesta de la Oficina: "La Avenida 9 de Julio con sus $140 \mathrm{~m}$. de ancho parecerá exageradamente ancha para el que no la considere como tramo central de penetración de una arteria maestra de tráfico regional" (57), afirmaba en 1937, depositando en el proyecto una frustración, la imposibilidad de constituir un organismo para la aglomeración bonaerense. Llevar a cabo una obra a escala con la magnitud del "gran Buenos Aires", sustituía la necesaria coordinación metropolitana. Con idéntica argumentación, el megaproyecto se vislumbra como una respuesta de zonning, aumentando los espacios libres y verdes, solucionando los acuciantes problemas de la circulación y el estacionamiento de vehículos, y considerando la previsión, pieza clave de un Plan de Urbanismo. Todas las dimensiones del urbanismo se aunaban hipotéticamente en el proyecto. Pero, más que un resultado del diagnóstico preliminar (las razones previas que indicarían la conveniencia de esa obra por sobre la factibilidad de otras), el diagnóstico es posterior y es utilizado en tanto justificación del emblemático proyecto del treinta.

Concretamente, esta Avenida, inspirada junto con la Av. de Mayo en las operaciones de apertura decimonónicas, será aprobada legalmente en los inicios del siglo y reformulada por los sucesivos documentos de urbanismo. Inclusive, el Plan Director, elaborado por Kurchan y Ferrari Hardoy en el taller parisino de Le Corbusier, esa ville radieuse para Buenos Aires, tomará como eje estructurante la Av. 9 de Julio (58). La obra se inició en 1937 con cuatro cuadras y creció espasmódicamente hasta concluirse en 1996, como parte de la red de autopistas metropolitanas, proyectadas por los gobiernos militares. A lo largo del siglo, el proyecto recibe la impronta de las ideas urbanísticas de cada escenario histórico.

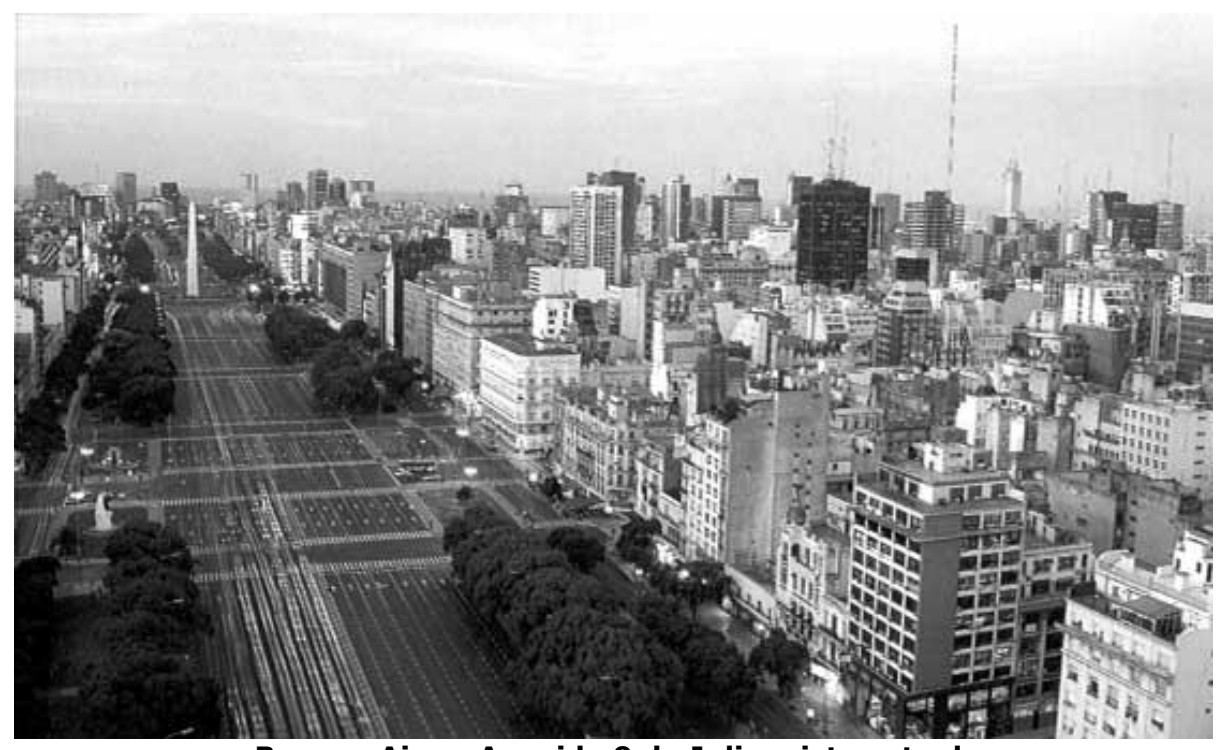

Buenos Aires, Avenida 9 de Julio, vista actual.

Tarjeta postal en archivo M.I.P.R. Reproducción parcial en blanco y negro con fines estrictamente culturales. 


\section{Planes y Proyectos}

Si revisamos los "casos" que venimos de presentar es posible identificar la emergencia del urbanismo moderno. En el Nuevo Plano (1909), el Plan se define como "una serie de reglas generales para que las autoridades se ajusten en la ejecución de las obras en el tiempo". En el formato de un "plan de embellecimiento y extensión" se limitó a resolver el trazado y a establecer los límites entre lo público y lo privado, desde una idea de ciudad cerrada y jerarquizada. Los focos de la composición fueron los proyectos de espacios públicos y los edificios representativos del Estado Nacional. La justificación, (aún no tiene la dimensión de un diagnóstico) se efectúa en función de los instrumentos compositivos.

La introducción del "diagnóstico" desde los "métodos de la ciencia positiva" y el interés en considerar todas las dimensiones de los problemas de la ciudad, se plantean in nuce con el Urbanismo Moderno. En ese momento, el rol asignado a los estudios de la evolución urbana y a los estudios preliminares, se consideran como fundamentos del diseño. No obstante se instala un conflicto entre lo estudiado y lo proyectado, entre la consideración del conjunto de la ciudad y sus partes, que no termina de resolverse. Dar forma, problema clásico que la arquitectura entra en colisión con los lenguajes científicos implementados en los estudios. Esas tensiones se manifiestan en el Proyecto Orgánico (1923-25) se intentan anular en la propuesta de ciudad azucarera (1924)y más tarde en el Plan Director, que elaboran Kurchan y Ferrari-Hardoy.con Le Corbusier en Paris (1938). Al igual que la gran mayoría de los planteos de entre-guerras en América y en Europa, los planes no se llevan a cabo. Sin embargo, sus ideas toman materialidad a través de las intervenciones fragmentarias. Podría aducirse que este texto focalizado en el período genético del urbanismo, pone énfasis en los principios tributarios del arte urbano, que se pierden en el pasaje del urbanismo a la planificación. Cuando la idea de ciudad se extiende a la Región, cuya "forma" se diluye en la abstracción de los planteos del zonnig y las reglamentaciones. ¿Acaso esa lógica no persistió en las décadas de posguerra? 


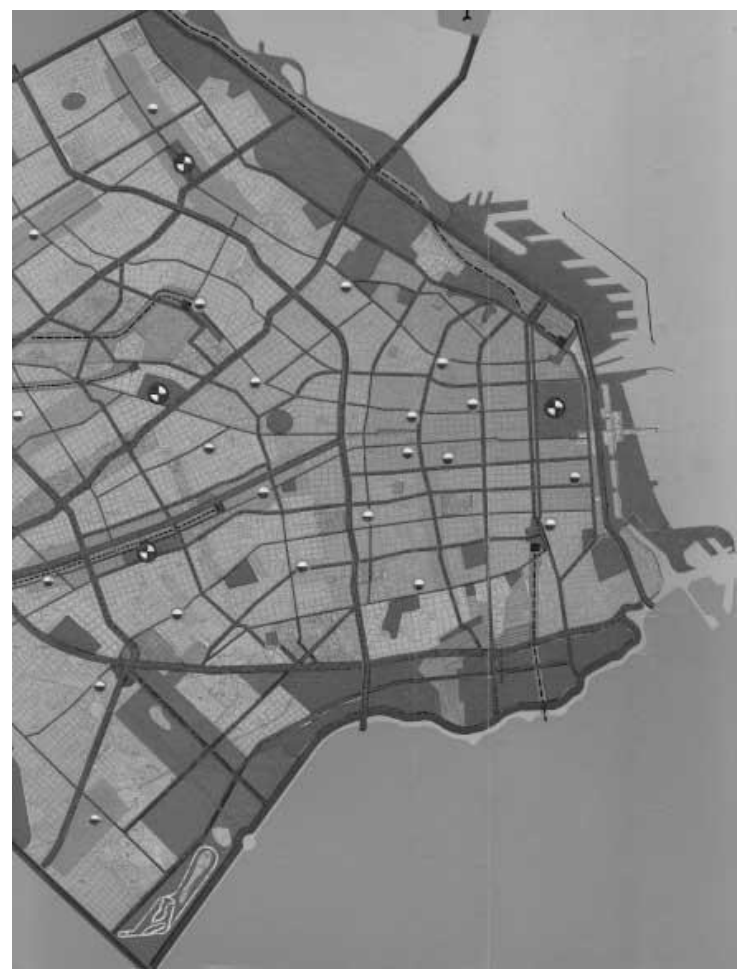

Plano Director de Buenos Aires, sección. Plano que incorpora diversas iniciativas previas de proyectos del Ing. Della Paolera y Le Corbusier, entre otros. Reproducción parcial, en blanco y negro, a partir de plano color en: MUNICIPALIDAD DE LA CIUDAD DE BUENOS AIRES,

Organización del Plan Regulador. Buenos Aires: Informe Preliminar. Etapa 1959-1960, p. 243.

Por gentileza del Arqto. Alberto Gurovich W., en Santiago de Chile. 
Los planes modernos, revelan las formas de pensar la ciudad en un momento dado, las intenciones globales sobre su sociedad y su espacio. Asimismo, las partes de planes que se concretan son aquellos "proyectos" consensuados que persisten en el tiempo (59). Estos siempre interactuaron con la ciudad existente, con sus espacios indeterminados y sus tiempos largos. Los tiempos de las concepciones originales y las reinterpretaciones técnicas, aquellos de las decisiones políticas, de la memoria y de las prácticas sociales.

Tal como reseñamos al principio, el "proyecto urbano" es una noción reciente. Sus características responden a un escenario histórico preciso de globalización, de ponderación de partenaires privados, cuando los estados se achican y pierden presencia en la producción de la ciudad. Sin embargo, en ellos persisten muchos de los conflictos constitutivos que históricamente se planteó el urbanismo.

\section{Referencias}

\section{Citas y Notas}

1. Devillers, Christian, "Le projet urbain", Conférences Paris d Árchitectes, Pavillon de I'Arsenal, Paris, Editions du Pavillon de I'Arsenal, 1994. Cfr. también, Efron, Jean-Pierre (sous la direction de), Les architectes et le projet. Tomes 1 et 2. Architecture, Une methodologie. Institut Français d'Architecture, Pierre Mardaga, Liège, 1992.

2. Lacaze, J.P, Les méthodes de I'urbanisme, PUF, Paris, 1993, pp. 18-19.

3. "La aparición de términos nuevos o la difusión de términos antiguos bajo nuevas acepciones marcan generalmente la emergencia de problemáticas nuevas cuyo estudio puede dilucidar". En Choay, Françoise, "Conclusion", in Merlin, P.(ed.), Morphologie urbaine et parcellaire, Espaces, St. Dennis, 1988, p.25.

4. "Es el procedimiento por el cual, mediante el uso de instrumentos especificos, se llega a la predeterminación sintética, a la formación y a la ejecución de obras arquitectónicas en el interior de las aglomeraciones urbanas, se trata de un procedimiento mediante el cual una serie de datos objetivos y de problemas abstractos de situaciones culturales y exigencias sociales se diferencian encontrando una expresión morfologicamente orgánica y linguisticamente unitaria". En Porthogesi, P., Dizionario Enciclopedico de Archittetura e Urbanistica, Roma, Instituto Editoriale Romano, 1969.

5. Tecnologías Urbanas Barcelona SA, Guía para la puesta en marcha de un Plan Estratégico, Barcelona, 1994.

6. Ascher, François, "Projet public et réalisation privées. Le renouveau de la Planification des villes", Les Annales de la Recherche Urbaine Nro. 51, 1993.

7. Gaudin,Jean-Pierre, Desseins de villes.Art Urbain et Urbanisme, Paris, L'Harmattan, 1991.

8. En referencia a las propuestas de Camillo Sitte, Collins se preguntaba: "es el modo de análisis y planeamiento sittesco algo dinámico que aflora de vez en cuando en la historia de forma cíclica o es unicamente un fenómeno moderno, la búsqueda de una estética urbana retrospectiva que solo sobrevive en 
http://revistaurbanismo.uchile.cl

Planes versus proyectos:

Algunos problemas constitutivos del Urbanismo Moderno. Buenos Aires (1910-1936).

el viejo mundo (...)?. Collins, G, "Re-consideración de Camillo Sitte", en Collins G y Colllins, C, Camillo Sitte y el nacimiento del urbanismo Moderno,Ed. Gustavo Gilli, Barcelona, 1980. p.145.

9. Novick, Alicia, "Técnicos locales y extranjeros en la génesis del urbanismo porteño". en AREA, Revista de Investigaciones. FADU-Escuela Politécnica de Laussane. Diciembre de 1992.

10. A nivel nacional se constituye en 1894 la Comisión para el Estudio y Reforma del Departamento de Ingenieros que como corolario de su trabajo determina la necesidad de crear el Ministerio de Obras Públicas (1989) En 1912 se crea la empresa Obras Sanitarias de la Nación. A nivel municipal, en 1892 se dicta el Reglamento General de Reparticiones que delimita las competencias entre dependencias y su organización interna.

11. En los salones de la Sociedad Científica Argentina se decide la creación de las principales Asociaciones que se ocupan de la ciudad. La Sociedad Central de Arquitectos (1901), El Museo Social Argentino (1912), etc. Los higienistas dispusieron de otros espacios, pero para los arquitectos, y sobre todo para los ingenieros, la Sociedad Científica fué el sitio de debate y reflexión privilegiado hasta el momento en que crearon sus respectivas corporaciones profesionales.

12. Silva, Ángel, "El catastro, su conservación", Anales de la Sociedad Científica Argentina N 6, Buenos Aires, 1878. Soulanges, Edmundo "Método racional para el relevamiento de un plano catastral por el método de poligonación, observaciones sobre el plano catastral de la Municipalidad". Anales de la Sociedad Científica Argentina, N. 40, Buenos Aires, 1895.

13. Belgrano, Joaquín, Rojas Félix, "Estudio del informe crítico sobre el proyecto de reglamentación de construcciones para la ciudad de Buenos Aires, confeccionado por la Oficina de Ingenieros Municipales". Anales de la Sociedad Científica Argentina, N²1, Buenos Aires, 1886.

14. Buschiazzo, Juan A, White, Guillermo, Huergo, Luis, Otamendi, Rómulo, Blot, Pablo,"Informe sobre pavimentación de la ciudad". Anales Sociedad Científica Argentina, № 27, Buenos Aires, 1889.

15. Con respecto a las transformaciones en las instituciones de la higiene pública, cfr. Paiva, Verónica, Higienismo y ciudad, Informe de avance, Beca de iniciación UBA. Buenos Aires, 1995.

16. En lo que hace al control edilicio, el Reglamento de Construcciones es un primer hito, en la medida en que establece normas a asigna responsabilidades respecto a una amplia gama de problemas que con anterioridad estaban a cargo de las Comisiones Auxiliares de vecinos. En 1901, se constituye la Inspección General en la Municipalidad. Cfr. Novick, Alicia, Nuñez, Teresita, "Mapa institucional", en De los Planes de embellecimiento a los planes estratégicos, Documento de trabajo CONAMBA, Buenos Aires, 1995.

17. "Edilidad. Mejoras de Buenos Aires", La Ingeniería. Órgano oficial del Centro Nacional de Ingenieros. No 25, Año III, Buenos Aires, 15 de junio de 1899.

18. Morales, Carlos María, "Algunos datos relativos al trazado general del Municipio", Anales de la Sociedad Científica Argentina No 46, Buenos Aires, 1898.

19. Sitte rechaza la cuadrícula americana de plano, asociándola a "la falta de programa" de países "sin pasado ni historia (y) que no podrían figurar en la historia cultural de la humanidad". Stuben defiende las ventajas higiénicas de la línea recta pero la condena desde el punto de vista artístico. La crítica a la monotonía de los trazados rectilíneos es retomada también por el Nuevo Plano desde la especulación salvaje, que los impulsa: "Si a lo menos esa obra hubiese sido concebida racionalmente, sea en vista de favorecer el tráfico, sea para obtener un efecto pintoresco y grandioso. Pero nada de ello. La única preocupación que haya guiado a los autores de ese plano ha sido combinar la partición más favorable para la venta de terrenos." 
20. Gorelik, Adrián, "La máquina reformista: la cuadrícula, el Estado y la emergencia urbanística de Buenos Aires, 1898-1909", Seminario Internacional Vaquerías, Documento de trabajo No 1, Córdoba, Argentina, 1996.

21. Sobre ese tema, cfr. Berjman, Sonia, Plazas y parques de Buenos Aires: La Obra de los paisajistas franceses. 1860-1930, Fondo de Cultura Económica, Buenos Aires, 1997.

22. Morales, Carlos María, "Las mejoras edilicas de Buenos Aires. Memoria presentada al Segundo Congreso Científico Latinoamericano de Montevideo", Anales de la Sociedad Científica Argentina, No 51, Buenos Aires, p. 181.

23. Sobre este tema cfr. Shmidt, Claudia, "Francisco Tamburini. Edificios para una Capital permanente", Cuadernos de Historia N 10, SCA-IAA, Buenos Aires, en prensa.

24. "La Avenida de Norte a Sur", Revista Municipal, 1895, p. 2028.

25. "Cuestiones locales. Paris-Buenos Aires", Revista Municipal, 1895, p. 1592.

26. Sitte, Camillo, L'Art de batir les villes. L'urbanisme selon ses fondements artistiques. V. Vincent, L'Equerre, Paris, 1980. (1ra.ed. 1889), p.131.

27. Cfr. "Notas sobre Planes y proyectos. Buenos Aires 1907,1909,1911", Buenos Aires. Territorio. Espacio Público y Profesionales de la Ciudad (Siglos XVIII al XX) en Serie: Escritos del Instituto de Arte Americano .IAA-Secretaría de Investigaciones de FADU-UBA, 1998.

28. Sobre Bouvard en Buenos Aires cfr entre otros: Berman, op.cit.y Tartarini, J, "El Plan Bouvard para Buenos Aires (1907-1911). Algunos antecedentes", ANALES N 27-28, IAA-FADU-UBA, Buenos Aires, 1992, Hardoy, Jorge,"Teorías y prácticas urbanísticas en Europa entre 1850 y 1930 . Su translado a América Latina", Jorge E. Hardoy y Richard M. Morse (Comp), Repensando la ciudad de América Latina. Buenos Aires, Grupo Editor Latinoamericano, Buenos Aires, 1988.

29. Intendencia Municipal, El Nuevo Plano, Buenos Aires, 1909, p.5.

30. Cloquet, Louis, La construction des villes. Conférence donnée le 11-1-1904 à la Section Gantoise de I'association des Ingénieurs sorties des écoles spétiales de Gand. Mons.Duquesne-Masquillier et fils,1904. (extr. Annales de I'Association des Ingénieurs sorties des Ecoles Spéciales).

31. Intendencia Municipal, El Nuevo Plano, Buenos Aires, 1909, pag.17. La apertura al río, que en Buenos Aires es bloqueada por la construcción del puerto, es planteada por Maillart en su proyecto sobre la Plaza de Mayo (1907) y retomada por el mismo Bouvard en su explanada de 1910. Esta idea de partido es tributaria de un proyecto posterior a la Exposición de 1900, que Bouvard defiende en Paris: "je compte amenager I'explanades des Invalides, les Champs de Mars et les berges de la Seine. Je voudrais créer, sur ces berges, des jardins et des terrases qui egayeraient un peu ce que ont de severe les remparts de pierre qui forment les quais. On n'a pas assez tiré parti de la Seine, la ligne du fleuve est d'une variété et d'un pittoresque qui permettent des jolis motifs de déco. Les jardins que $j$ 'entrevois relieraient le Trocadero au Champs de Mars", Le Temps, 17 juin., 1904, p. 458.

32. Intendencia Municipal. El Nuevo Plano...., op. cit., p.17-18.

33. Ibidem, p.22.

34. Intendencia Municipal, Comisión de Estética Edilicia, Proyecto orgánico para la urbanización del municipio. Buenos Aires, Buenos Aires, Talleres Peuser, 1925. Acerca de este plano, cfr. Berjman, op. cit., 
http://revistaurbanismo.uchile.cl

Planes versus proyectos: Algunos problemas constitutivos del Urbanismo Moderno. Buenos Aires (1910-1936).

Gorelik, Adrián, "La búsqueda del centro. Ideas y dimesiones de espacio público en la gestión urbana y en las polémicas sobre la ciudad: Buenos Aires 1925-1936", Boletín del Instituto de Historia Argentina y Americana "Dr Emilio Ravignani", Nro. 9, Tercera Serie, Buenos Aires, 1994.

35. Intendencia Municipal. Comisión ....op. cit, p.362.

36. Ibidem, p.60.

37. Ibidem, p. 36.

38. Ibidem, p. 362.

39. Ibidem, p.274.

40. Ibidem, p.287.

41. A propósito de los protagonistas del Arte urbano europeos, se efectúa una caracterización que cabe a los miembros de la Comisión de Estética edilicia: "los protagonistas de esta cultura vivieron la angustia de la modernidad, Aceptación del cambio y rechazo de los cánones estéticos que conlleva, reivindicación de un sentimiento de pertenencia, noción del "estilo nacional" como de un valor a defender. Un testimonio significativo del conflicto profundo vivido por una generación que duda entre la conciencia de lo nuevo, la apropiación del progreso, la salvaguarda o la valorización del pasado". Calabi, Donatella, "Dilettantes on le devient", In Smets, M, Charles Buls, Bruselas, Mardaga, 1994.

42. Intendencia Municipal, Comisión ...., op. cit., p.362.

43. Vautier, Ernesto, Prebisch, Alberto, "Ensayo de Estética Contemporánea" , Revista de Arquitectura, Buenos Aires, 1924, p.110.

44. Ibidem, p.108.

45. Intendencia Municipal. Comisión..., op.cit., p.361.

46. Ibidem, p.362.

47. Della Paolera, Carlos María, Contribution à l'étude d'un plan d'aménagement, d'embellisement et d'extension de Buenos Aires. Etude sur l'évolution de la ville. Tesis presentada en noviembre de 1927 en el Institut d'Urbanisme de Paris. Presidente del Jurado: Marcel Poète.

48. Della Paolera, Carlos María, " El Plan Regulador de la "Aglomeración Bonaerense. 1.La Capital y las zonas edificadas exteriores a su juridicción ", La Razón, 14 de abril de 1927.

49. Oficina del Plan de Urbanización, El Plan Regulador para Buenos Aires, mimeo, s-f. Tasmbién el Boletín del Honorable Concejo Deliberante No 39-40, 1943, presenta un listado completo de las obras efectuadas por la Oficina dirigida por Della Paolera.

50. Cfr. Novick, Alicia, Piccioni, Raúl, "Carlos María della Paolera o la amnesia del urbanismo argentino", Anales IAA, No. 29, Buenos Aires, 1998.

51. Bereterbide, Fermín, "Proyecto de ejecución de la avenida transversal de Norte a Sud. Formación de los Centros Cívicos Nacional y Municipal. Avenidas a bajo nivel y playas subterráneas de estacionamiento de automóviles." En Revista de Arquitectura, Septiembre de 1932. 
$\mathrm{http://revistaurbanismo.uchile.cl}$

Planes versus proyectos: Algunos problemas constitutivos del Urbanismo Moderno. Buenos Aires (1910-1936).

52. Respecto del proyecto de Saarinen, cfr. Ciucci, G, Dal Co, F, Manieri-Elia, M, Tafuri, M, La Ciudad Americana, de la guerra civil al New Deal, Ed. Gustavo Gili, Barcelona, 1975, p. 429.

53. Vautier, Ernesto, "La Reglamentación de las Construcciones en la Av. Norte-Sur. Organización del Volumen edificado del loteo y del trazado circulatorio", Revista de Arquitectura, junio de 1933.

54. Cabe citar como ilustración el Primer Congreso Internacional de Urbanismo Subterráneo realizado en Paris en julio de 1937.

55. Guido, Ángel, Monumentalización funcional de la Av. 9 de Julio de Buenos Aires, Conferencia pronunciada en el salón de Actos del Concejo Deliberante el 25 de abril de 1941 bajo los auspicios de "Los Amigos de la Ciudad".

56. Cfr. entre otros, "La Avenida 9 de Julio", Revista Municipal de Engenharia, Río de Janeiro, 1937. Dirección del Plan de Urbanización, 2da. Exposición Municipal de Urbanismo, Buenos Aires, 1939.

57. "La Avenida de Norte a Sur", La Prensa, 5 de mayo 1937.

58. El documento elaborado en Paris en 1938 se publica fragmentariamente en las Obras Completas de Le Corbusier y en su totalidad como "Plan Director para Buenos Aires", Versión Castellana de la Arquitectura de Hoy, Ed. Kraft, Buenos Aires, abr. 1947.

59. Según hipótesis recientes, en la composición del plano, se perfila un consenso sobre las ideas técnicas acerca de la ciudad pero también se "fija" y da forma a las ideas corrientes. Desde ahí los urbanistas son "reveladores" de las intenciones sobre la ciudad, pero también "fijadores". Las partes del proyecto que se traducen en proyectos urbanos serían aquellas que consensuadas persisten estables en el tiempo. LORTIE, André (1992), "Dessins de villes et destins de plans". Quels dess(e)ins pour les villes?. De quelques objets de planification pour I'urbanisme de l'entre-deux guerres?. Direction de la Recherche et des Affaires Scientifiques et Techniques, Paris, 1992. 